\title{
Experiencing the future: the influence of self-initiation on temporal perception
}

TIMOTHY VERSTYNEN, MICHAEL OLIVER, AND RICHARD B. IVRY

\begin{abstract}
Summary
Anticipation is a hallmark of skilled movements. For example, when removing plates from a loaded tray, the upward force generated by the supporting hand is reduced in anticipation of the reduced load. An adjustment of the postural force occurs as a result of the predicted consequences of the self-initiated action. Although the effect of anticipatory processes is easily discerned in the actions themselves, it is unclear whether these processes also affect our perceptual experience. In this chapter we focus on the relationship between action and the perceptual experience. We begin by reviewing how actions provide reliable predictions of forthcoming sensory information. Following this, we discuss how the anticipation of the time of external events is an important component of action-linked expectations. Finally, we report two experiments that examine how temporal predictions are integrated with the incoming sensory information, evaluating whether this integration occurs in a statistically optimal manner. This predictive process provides the important advantage of compensating for lags in conduction time between peripheral input and the central integration of this information, thus overcoming the physical limitations of sensory channels.
\end{abstract}

\subsection{Racing against sensory delays}

An important problem for the brain to solve is how to compensate for the temporal gap between when a stimulus is registered by a sensory detector and when it is recognized, either consciously or subconsciously, in the cortex. In humans, such delays happen on the order of hundreds of milliseconds (for review, see Welch \& Warren 1986). This slow conduction time can greatly impair how we react to events in our local environment. In many cases, this can mean the difference between smooth, coordinated actions and clumsy gestures. For example, reaching movements would be inherently unstable if the final position were achieved solely on the basis of visual and proprioceptive feedback signals. Such delays would invariably lead to significant endpoint oscillations (Lacquaniti et al. 1982; Soechting et al. 1986).

The negative effects of sensory lags can be attenuated by maximizing the efficiency of the input channels. Such an approach has been applied in the field of robotics with great

Space and Time in Perception and Action, eds. Romi Nijhawan and Beena Khurana. Published by Cambridge University Press. (C) Cambridge University Press 2010. success

limits th true inst: existing be enhan Second, between

There anticipat and whe respond of the vi in its rec which w veridical. when cor (Nijhawa our exper are gener an object weight ill perceived

\subsection{The}

Action-lin postural a table of $d$ each plate expected each plate generated each succe 1985; Lun intimately cues for th patron assi the resistiv displaceme

Does the The many a relations 
success (Blomdell et al. 2005). Unfortunately, the physiology of the human nervous system limits the speed of conduction of sensory signals. Therefore, the brain must estimate the true instantaneous state of the world by adopting a strategy of predictive inference based on existing sensory information. This has several advantages. First, perceptual processing can be enhanced by focusing attention to environmentally relevant events before they happen. Second, it can increase the reactive speed of our actions by compensating for the delays between the activation of sensory receptors and central processing.

There are many ways by which the brain extrapolates from current information to anticipate future sensory events. Spatiotemporal regularities are exploited to predict when and where a stimulus will occur. For example, neurons in the primary visual cortex will respond more vigorously to a visual stimulus that is repeatedly presented in the same part of the visual field and at predictable intervals than to a stimulus that randomly appears in its receptive field (Sharma et al. 2003). Motion extrapolation is another method by which we predict the future location of a moving object. Such processes need not be veridical. For example, a moving stimulus is perceived as being further along its trajectory when compared to a stationary object, even if the two objects are at the same location (Nijhawan 1994). We also make use of general physical principles or intuitions, based on our experience of consistent environmental features. For example, because larger objects are generally heavier than smaller objects we make a priori inferences about the weight of an object based on its size. This prediction also can distort our perception, as in the sizeweight illusion (Charpentier 1891), whereby the smaller of two objects of equal weight is perceived as the heavier of the two.

for the temporal gap it is recognized, either ys happen on the order . This slow conduction snment. In many cases, s and clumsy gestures. the final position were k signals. Such delays ti et al. 1982; Soechting

mizing the efficiency of d of robotics with great

\subsection{The influence of action on perception}

Action-linked expectations manifest themselves in many of our movements. Anticipatory postural adjustments constitute one class of such actions. Consider the waiter arriving at a table of diners with a tray full of entrees. His ability to keep the tray steady as he serves each plate is quite impressive given that more than $100 \mathrm{msec}$ of processing delays can be expected before his brain will register the change in the downward force that occurs as each plate is lifted. Stability is maintained by a predictive reduction of the upward force generated by the supporting hand against the tray. This reduction must occur just before each successive plate is removed by the active hand (Hugon et al. 1982; Dufossae et al. 1985; Lum et al. 1992; Masson et al. 1999). These anticipatory predictions appear to be intimately linked to the waiter's self-generated actions, as they are not observed when the cues for the unloading are only sensory in nature (Diedrichsen et al. 2003). If a helpful patron assists by reaching over and taking her own entrée, an unfortunate mess may occur. If the resistive force generated by the supporting hand remains too large, the resulting upward displacement of the tray may launch the remaining plates into their individual orbits.

Does the anticipatory response of the waiter also influence his perceptual experience? The many recursive connections between motor and sensory areas in the brain suggest a relationship of mutual influence (for review, see Nelson 1996). Indeed, there are many 
demonstrations of situations in which our actions influence our perceptions. A classic example involves the "tickling effect" (Weiskrantz 1971). If someone brushes the tip of their finger across the palm of your hand, the tactile sensation can be quite ticklish. However, when you use your other hand to create a near-identical tactile stimulus, the ticklish sensation is attenuated. Thus, the sensory experience is more intense when it results from the actions of an external agent compared to when it is self-produced. The attenuated experience from self-stimulation results from our ability to precisely predict, in space and time, the tactile stimulus (Blakemore et al. 1999). In the self-stimulation condition, the insertion of a slight delay between the participant's own movement and the resulting tactile stimulus will lead to an increase in the intensity of the tactile experience. Altering the angle between the direction of the movement and the tactile stimulus will also increase the perceived intensity. Thus, the attenuation of ticklishness is maximized when the somatosensory experience on the palm of the hand matches the spatiotemporal profile of the action used to produce it.

Another powerful example of how action-linked predictions influence perception is the occuloparalytic illusion, described by Matin and colleagues (1982). After receiving injections of d-turbocurarinem, a cholinergic antagonist of the muscle spindles and extrafusal muscles that leads to the transient paralysis of eye movements, participants were asked to localize visual and auditory targets in space. Perceived location of external targets was greatly influenced by how the participants intended to move their eyes. Thus, action-based changes in visual perception can result from anticipatory consequences of motor commands to the eyes, even if those actions are not implemented.

How might a phenomenon such as the tickling effect or the occuloparalytic illusion extend to the sensory experience of our hypothetical waiter as he serves the dinner entrees? Is his perception of the tray's weight affected by his actions? Predictive mechanisms allow for anticipatory postural adjustments prior to the volitional actions required to remove each plate. But does this expectation also modulate his perceptual experience of the force imposed by the tray?

To examine this issue, we tested a group of participants in a modified version of the unloading task (Diedrichsen et al. 2007). Participants were situated in a 3D visual-haptic virtual reality environment where they could manipulate simulated objects. Participants were instructed to lift and hold a platform-like object with one hand (the postural hand; Fig. 11.1(a)). After a brief stabilization period, the virtual object was lifted, either by an action of the participant, self-unloading, or by an unseen agent, external-unloading. During self-unloading, a cue instructed the participant to use his or her other hand to lift the object as quickly as possible. On external-unloading trials, the forces acting on the object were programmed such that the object rose off the supporting hand in a manner that simulated the dynamics of self-unloading; the participant did not move the other hand on these trials.

As noted previously, self-unloading results in a reduced upward perturbation of the postural hand compared to external-unloading. This effect could occur because of a welltimed reduction in the upward force generated by the postural hand or by increasing the stiffness of the postural hand, perhaps by cocontraction of antagonist muscles. The virtual 
ptions. A classic brushes the tip of icklish. However, ticklish sensation ts from the actions d experience from ad time, the tactile nsertion of a slight timulus will lead to tween the direction red intensity. Thus, experience on the to produce it.

ce perception is the fter receiving injecndles and extrafusal sicipants were asked external targets was . Thus, action-based of motor commands suloparalytic illusion es the dinner entrees? ve mechanisms allow s required to remove iperience of the force

Codified version of the in a $3 D$ visual-haptic 4 objects. Participants and (the postural hand; was lifted, either by an nal-unloading. During th hand to lift the object wing on the object were manner that simulated other hand on these

yard perturbation of the accur because of a welland or by increasing the hist muscles. The virtual (a)

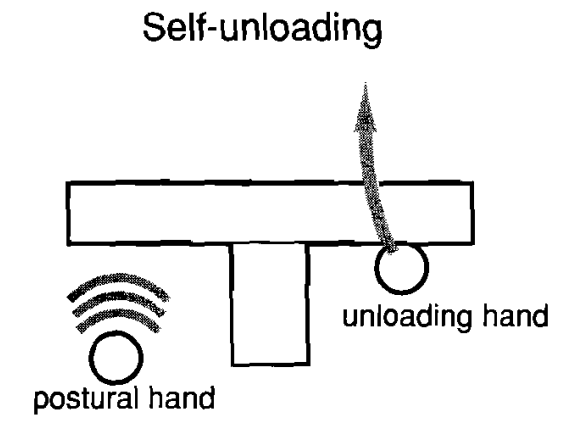

\section{External-unloading}

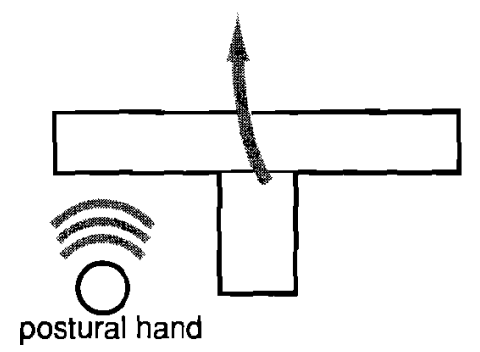

(b)

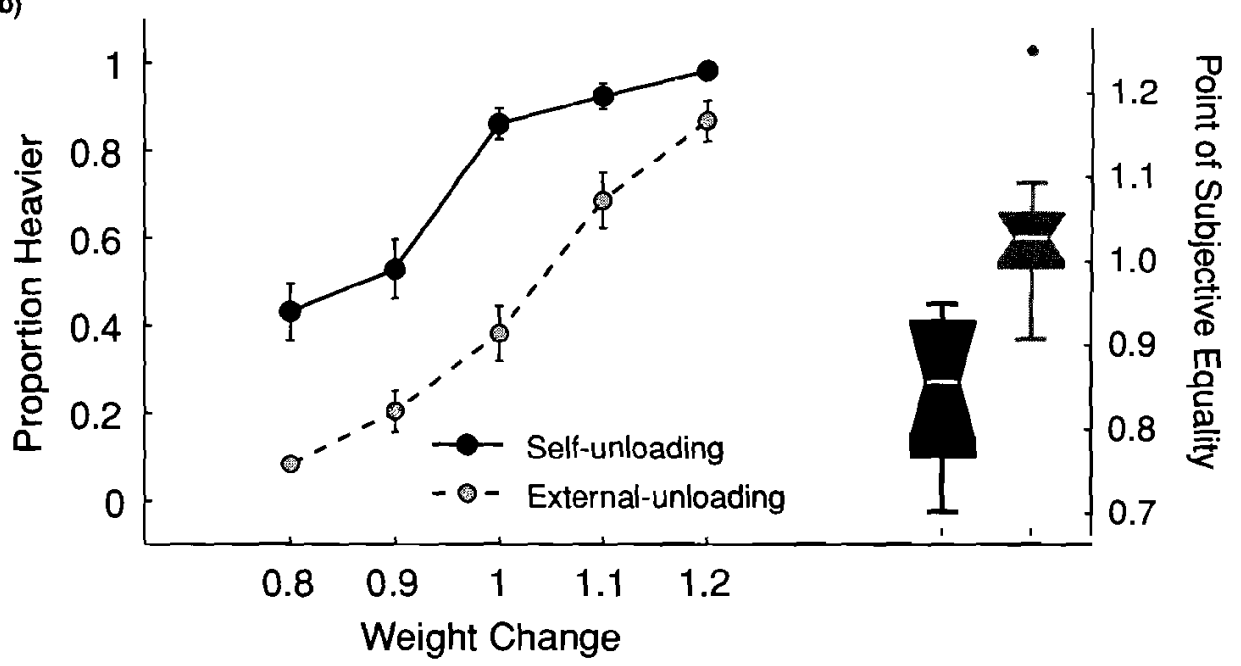

Fig. 11.1 Influence of action on force perception. (a) Participants were instructed to lift and hold a virtual object with one hand. Following a cue, participants had to either unload the object using the other hand (self-unloading) or observe the computer perform the lifting action (computer-unloading). On most trials, a "phantom" force that remained on the loaded hand was adjusted at the time of unloading to be heavier or lighter than the force of the object prior to unloading. (b) Psychometric functions of perceived force changes and point of subjective equality (PSE) estimates. Participants perceived the phantom force as being heavier during the self-unloading trials than during the computerunloading trials. (Reprinted from Diedrichsen et al. 2007.)

reality environment can be exploited to contrast these two mechanisms by the inclusion of "catch" trials (Diedrichsen et al. 2003, 2005). On some of the self-unloading trials, the visual feedback, as well as haptic experience of the unloading hand, cues the participant that the object is being lifted from the postural hand. However, the downward force generated by the virtual object remains on the postural hand. A downward deviation of the postural 
hand is observed during these catch trials, indicating that the anticipatory response is in fact due to a reduction in the upward force rather than an increase in stiffness.

The "phantom" force used to create the catch trials can also provide a probe on whether perception is altered by the anticipatory mechanisms. Following self-unloading trials, we asked participants to judge the haptic experience of the postural hand. The initial force of the phantom objects was $3.5 \mathrm{~g}$. Participants were told to report whether a force remained on the postural hand (as would be true on catch trials) or was absent (as would be true on natural unloading trials). On the catch trials, we altered the force of the object right at the time of unloading, with the resulting force ranging from $80-120 \%$ of the original $3.5 \mathrm{~g}$ force. By varying the phantom force, we could ask participants to not only report whether it was present or absent, but also whether, when present, it had "increased" or "decreased" in comparison to the force experienced prior to unloading. ' For comparison, we also included external-unloading trials. Here, the object was seen to rise off the postural hand and, with similar probabilities, the force on that hand was either removed or persisted in a similar manner as on self-unloading trials.

Figure 11.1(b) shows the psychometric functions for the self- and external-unloading trials. On catch trials, participants consistently perceived the phantom force as greater during self-unloading trials compared to external-unloading trials. The point of subjective equality (PSE), defined as the force at which a participant is equally likely to judge the force as "increased" or "decreased," was significantly different between the two conditions $(t(11)=5.51, p<0.001)$. Moreover, during self-unloading, the participants were biased to perceive the phantom force as having increased in comparison to the force experienced prior to unloading. On trials in which the force was unchanged, participants reported an increase of force on approximately $85 \%$ of the trials. We assume that the bias on selfunloading trials reflects a comparison process in which the sensory experience is compared to a representation that incorporates anticipated changes in the sensory experience due to self-generated actions.

This experiment suggests that our hypothetical waiter does, in fact, perceive the changes in the weight of the tray differently depending on whether he removes the plates himself or whether he is assisted by the eager patron. Whether reconstructing the visual environment (Matin et al. 1982), experiencing a ticklish tactile stimulus (Weiskrantz 1971; Blakemore et al. 1999), or perceiving haptic forces (Diedrichsen et al. 2007), our actions lead us to predict forthcoming changes in sensory information and influence our internal perceptual reconstruction of the environment.

\subsection{Predicting the future}

Predictive mechanisms anticipate sensory events and can compensate for delays in the conduction of sensory information from the periphery to the brain. This not only helps

\footnotetext{
I Although our main interest is in the perceived force on catch trials, it was necessary to include natural unloading trials because the anticipatory postural adjustment is rapidly extinguished by the catch trials (Diedrichsen et al. 2005). Thus, catch trials only occurred on $33 \%$ of the trials.
}

to $\mathrm{c}$

with

out ,

actic

ever

In

our

sinc

hanc

the

tick,

Cha]

were

$55 \mathrm{~d}$

"1."

ever

and

long

that

dura

som

chan

$\mathrm{T}$

in" $i$

chan

the s

duri

lost

First

audi

high

$\mathrm{Sec0}$

pres

$\mathrm{Al}$

links

stim

by $\mathrm{H}$

of $s$

case,

invol

or ar

The 
ory response is in finess.

probe on whether nloading trials, we The initial force of a force remained s would be true on object right at the the original $3.5 \mathrm{~g}$ ly report whether it " or "decreased" in n, we also included hral hand and, with rsisted in a similar

external-unloading m force as greater point of subjective likely to judge the the two conditions ipants were biased force experienced tipants reported an the bias on selftrience is compared experience due to

rceive the changes he plates himself or visual environment 2. 1971; Blakemore actions lead us to internal perceptual

for delays in the his not only helps

fll unloading trials because $05)$. Thus, catch trials only

to construct veridical percepts but facilitates stable and appropriate actions as we interact with the environment. Given that these internal expectations are, at least in part, produced out of a necessity to overcome temporal delays in the processing of sensory information, action-induced expectations may also modulate our perception of the timing of sensory events.

Indeed, Yarrow and colleagues (2001) reported evidence that actions can directly modify our perception of time. They set out to investigate a puzzling illusion that has been noted since the advent of the analog clock; namely, why does the initial tick of the seconds hand appear to move slower when first glancing at a clock than subsequent ticks? In the extreme, it is frequently reported that the clock appears to briefly stop after the first tick, an illusion referred to as chronostasis. This phenomenon is discussed in detail in Chapter 10, so here we will provide only a brief summary of the Yarrow study. Participants were required to saccade to a stimulus, the number " 0 ," that was positioned either 22 or $55 \mathrm{deg}$ from fixation. Once the saccade was initiated, the stimulus changed to the number "1." This number was present for a variable duration, and subsequently incremented by one every $1000 \mathrm{msec}$. Thus, the perception was of a seconds counter incrementing periodically and participants were asked to judge if the duration of the initial number was shorter or longer than the other numbers. Participants judged the duration of the " 1 " to be longer than that of the subsequent numbers, and this effect was influenced by the amplitude (and thus duration) of the saccade. In fact, the temporal distortion was so profound that participants sometimes judged that the onset of the " 1 " occurred prior to saccade, which triggered the change.

This result led the authors to hypothesize that chronostasis arises from the need to "fill in" information that is lost during the saccade. Because the movement triggers the stimulus change, the estimate of the stimulus onset gets linked to the motor command that generates the saccade. A key assumption here is that the visual system is blind to visual information during the eye movement. Although it remains to be seen whether visual information is truly lost during saccades, two pieces of evidence run contradictory to this lost-time hypothesis. First, the chronostasis effect is not limited to the visual modality, but is also present for auditory (Hodinott-Hill et al. 2002) and tactile events (Yarrow and Rothwell 2003). It is highly unlikely that all sensory modalities are "blinded" during saccadic eye movements. Second, chronostasis is also observed when the trigger for the stimulus change is a key press rather than a saccade (Park et al. 2003).

An alternative explanation for this modulation of temporal perception is that the brain links the motor command instigating the action with the onset of the resulting sensory stimulus. This hypothesis was suggested in a related set of elegant experiments conducted by Haggard and colleagues (2002). Participants were first asked to time the occurrence of several events by observing the rotating seconds hand on a clock. In the single-event case, participants were instructed to report the time of either a voluntary key press, an involuntary muscle twitch induced by transcranial magnetic stimulation of the motor cortex, or an external auditory click. Temporal judgments were veridical in all three conditions. The participants were then tested in an "operant" condition in which a tone was presented 
$250 \mathrm{msec}$ after each of the three events. The tone was perceived as occurring much earlier in the voluntary key press condition compared to the other two conditions. The authors suggest that the shift in the perceived timing of the tone is due to an association between the awareness of the voluntary action and its sensory consequence.

There is, however, one methodological concern with these studies. As noted previously, a moving object is perceived as being slightly ahead of its true state (Nijhawan 1994). This suggests that using the state of a moving clock as the dependent measure for time perception may introduce biases in estimating the timing of an event. Although it remains clear that temporal perception is influenced by self-generated actions differently than by other events (because the moving clock is the same for all conditions), the nature of the distortion is unclear because the dependent variable may reflect multiple processes. Moreover, the process by which a motor command influences the end sensory percept may be influenced by the attentional state of the participant in a different way than that which occurs in a purely perceptual condition. Spatial attention can alter the perceived temporal order of events as exemplified by the "shooting line" illusion (for review, see Schmidt 2000). It is possible that, when initiating an action, attention is briefly shifted to the appropriate area of space for the sensory consequence of that action. This shift may prime or alter the perceived onset of the sensory stimulus. Regardless of the underlying mechanism, the common finding from both the Haggard et al. (2002) and chronostasis studies is that voluntary actions appear to link the onset of the sensory percept with the motor commands used to instigate the actions themselves.

To further examine how the passage of time may be influenced by self-generated actions, we performed a series of experiments in which temporal judgments were made about static sensory events (Oliver et al. 2003). In the first experiment, eight participants were asked to compare the duration of two successive vibrotactile stimuli $(800 \mathrm{~Hz})$ presented to the middle finger of the left hand. The duration of one stimulus, the standard, was always fixed at $800 \mathrm{msec}$. The duration of the other stimulus, the comparison, varied from $600 \mathrm{msec}$ to $1000 \mathrm{msec}$. The order of the standard and comparison was randomized across trials. Following the second stimulus, the participant indicated which stimulus (the first or second) was longer in duration. The important manipulation involved the manner in which the stimuli were initiated. On each trial, the participant would initiate one stimulus by pressing a response key with the middle finger of the right hand. The onset of the other stimulus was controlled by the computer. The self-initiated stimulus could either be the standard or comparison stimulus, counterbalanced for the two orders. The computer presented a visual cue prior to either stimulus indicating whether a key press was required. For computer-triggered stimuli, the cue "Computer" was displayed and the vibrotactile stimulus began after a variable delay of 200-500 msec. For self-triggered stimuli, the cue "Self" was displayed. The vibrotactile stimulus began immediately after the subsequent key press.

The results from previously described studies suggest that voluntarily initiated sensory events are perceived as starting earlier than their true, veridical onsets. From this we predicted that the self-initiated stimuli would be perceived as having a longer duration than computer-initiated stimuli. As shown in Fig. 11.2, the results are consistent with this prediction. For a given duration, participants were more likely to judge the comparison 
ring much earlier ons. The authors aciation between

noted previously, hawan 1994). This or time perception remains clear that an by other events of the distortion ses. Moreover, the may be influenced occurs in a purely order of events as 900). It is possible te area of space for perceived onset of nmon finding from y actions appear to instigate the actions

f-generated actions, x made about static cipants were asked iz) presented to the $r$, was always fixed d from $600 \mathrm{msec}$ to d across trials. Fol(the first or second) aanner in which the stimulus by pressing e other stimulus was the standard or comresented a visual cue r computer-triggered us began after a vari' was displayed. The ss.

rily initiated sensory onsets. From this we ing a longer duration consistent with this judge the comparison

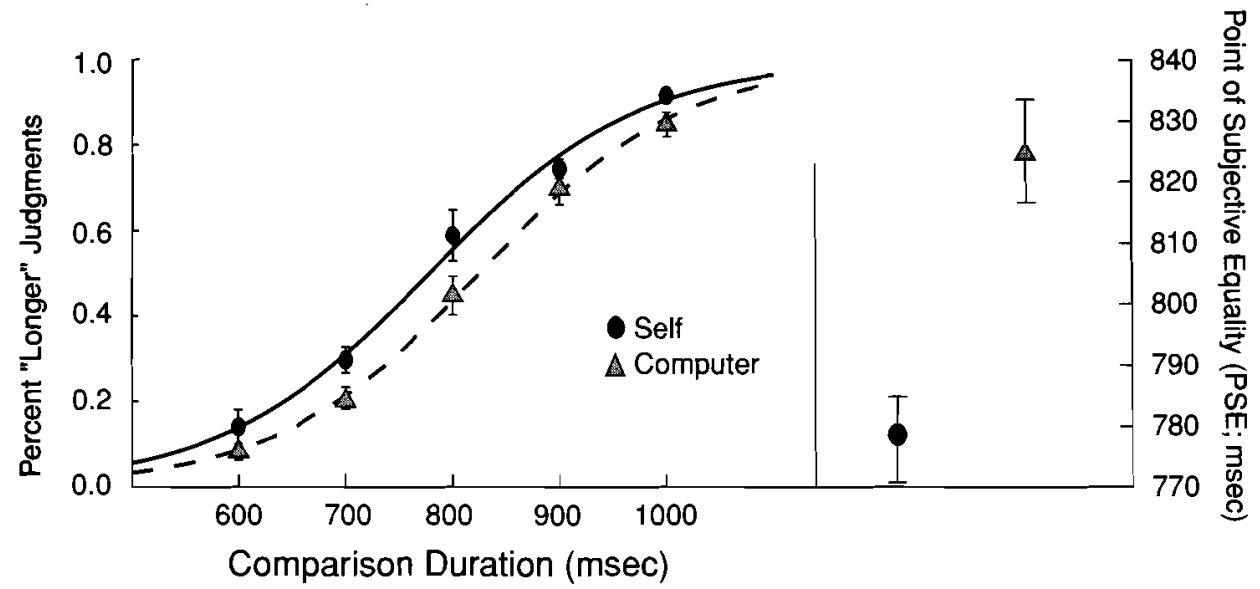

Fig. 11.2 Influence of self-initiation on vibrotactile perception. In a two-alternative forced choice experiment, participants were asked to compare the duration of a standard vibrotactile stimulus $(800 \mathrm{msec})$ to that of a comparison stimulus ranging in duration from 600 to $1000 \mathrm{msec}$ in steps of $100 \mathrm{msec}$. The stimulus was either initiated via a key press (self; black) or by an external agent (computer; gray). Participants consistently judged self-initiated comparison stimuli as being longer than computer-initiated stimuli of equal duration.

stimulus as longer for the self-initiated trials. This distortion is highlighted for trials in which the standard and comparison were both $800 \mathrm{msec}$. The self-initiated stimulus was judged "long" on approximately $60 \%$ of the trials when the two stimuli were, in fact, of equal duration.

To quantify this effect, we estimated the parameters of the psychometric function using binomial logistic regression

$$
\mathrm{y}=\lambda_{\mathrm{D}} \mathrm{x}_{\mathrm{D}}+\lambda_{0}
$$

where " $y$ " is the participant's binomial response on a given trial as to whether the comparison was longer or shorter than the standard and " $\mathrm{x}$ " is the duration of the comparison stimulus on that trial. The free parameters $\lambda_{\mathrm{D}}$ and $\lambda_{0}$ represent the weighted influence of changing stimulus duration on " $y$ " and the subject's own baseline response bias respectively. Assuming that the curves follow a logistic probability function, the point of subjective equality (PSE) is calculated as

$$
\mathrm{PSE}=-1 * \lambda_{0} / \lambda_{\mathrm{D}}
$$

If participants underestimate the perceived duration of the comparison stimulus, then the PSE should be greater than the actual standard duration (describing a psychometric function shifted to the right). In contrast, if participants overestimate the comparison's duration, the PSE should be smaller than the standard duration (e.g., shifted function to the left). The mean PSE value for trials in which the self-initiated stimulus was the comparison is $23 \mathrm{msec}$ less compared to when the self-initiated stimulus was the standard. 


\section{(a) No Delay Condition}

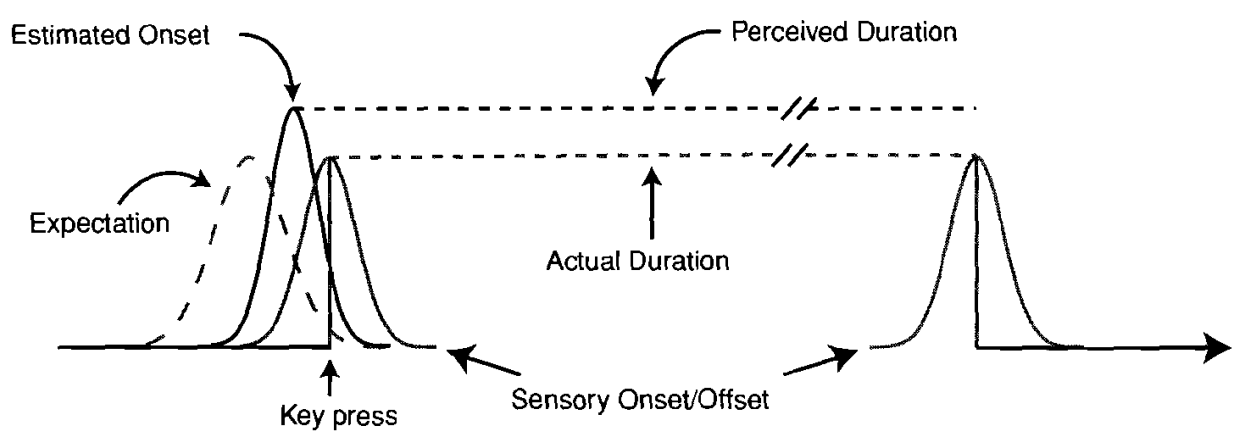

(b) $\mathbf{5 0}$ msec Delay Condition

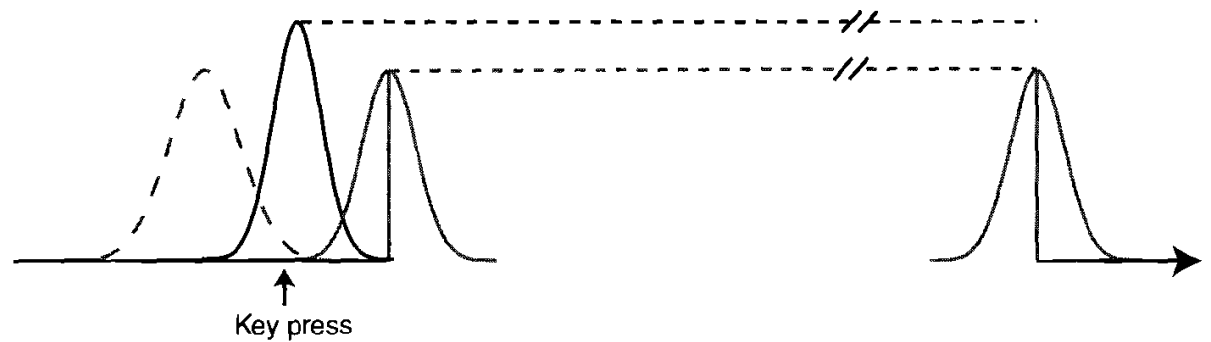

Fig. 11.3 Model in which self-initiated stimuli are perceived as being slightly longer than computerinitiated stimuli because of the incorporation of an a priori expectation generated by the motor command to trigger the stimulus. (a) Even when the stimulus is triggered immediately by the key press, the interval is perceived as longer because the perceived onset is a combination of the true sensory information and the action-linked expectation. (b) When a delay is inserted between the key press and the onset of the stimulus, the perceived onset ( $\mathrm{P}[$ onset]) is shifted farther away from the true onset ( $\mathrm{P}$ [haptic]). This is due to the influence of the action-linked expectation ( $\mathrm{P}[$ expect]), which remains locked to the motor command used to produce the key press.

To summarize, when a tactile stimulus on one hand is triggered by an action performed by the other, the perceived duration of that stimulus is lengthened compared to when the exact same stimulus is initiated by an external agent. Unlike the temporal distortion studies reported previously, participants in our study did not have to refer to an external clock; the judgments were based on internal mechanisms for representing temporal information. Nonetheless, the results are quite similar. It appears that the percept of the onset of a stimulus is attracted to a movement that initiated the stimulus. More importantly, the shift in perceived onset is pulled toward the point at which the intention to move occurs: that is, well before the action is initiated.

We propose that information linked to the motor command, for example, an efference copy signal or forward model, is used to anticipate the perceived onset of the stimulus. Figure 11.3(a) presents a schematic diagram of this hypothesis. Central to this model is 


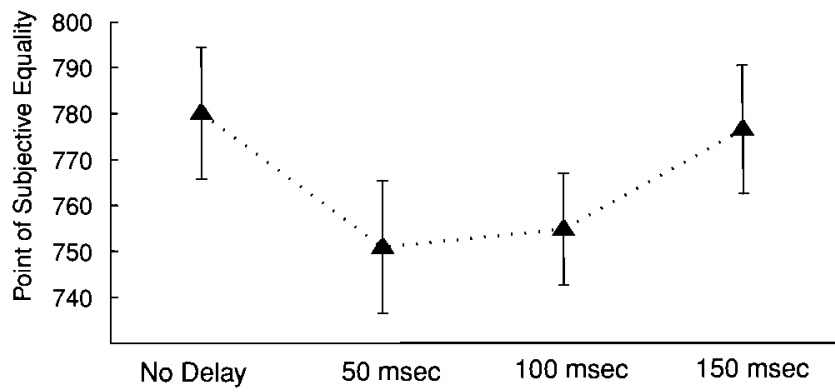

Fig. 11.4 Delay effects. Participants performed a temporal bisection task in which the onsets of all of the stimuli were triggered by a key press. Introducing a 50-msec delay between the key press and the stimulus onset resulted in an additional $30-\mathrm{msec}$ increase in the perceived duration of the stimulus. For longer delays, the illusion was reduced.

the idea that the perceived onsets and offsets of a stimulus (e.g., the vibrotactile stimulus in our study) are not veridical. Noise is introduced not only because of delays due to transmission along sensory channels but also because of variability in the transmission itself, decay of the signals within these sensory channels, and noise in the central decision processes. Self-initiated stimuli have an advantage in that the movement command help create an expectation of the forthcoming stimulus. The percept of the stimulus onset is thus a combination of a priori information linked to the motor command and the sensory information coming from the periphery. In contrast, there is no motor signal for computerinitiated stimuli to supplement the expected onset time. The expected probability function is simply a uniform distribution prior around the general time of the onset of the stimulus, because any point in time is equally as likely to be the onset as any other time around the key press.

We will further develop this model in the next section. First, we wish to explore one prediction derived from the basic ideas of the model. Introducing a small delay between the key press and the onset of the stimulus should lengthen the perceived duration of self-initiated stimuli. We tested this prediction in another group of ten participants using a temporal bisection task. An exposure phase was first run in which participants were presented examples of short $(600 \mathrm{msec})$ and long $(1000 \mathrm{msec})$ vibrotactile stimuli. Each example was self-initiated by a key press and followed by feedback indicating whether the stimulus had been short or long. Following this exposure phase, participants completed a series of self-initiated trials in which a single stimulus was presented for a variable duration and the participant categorized it as short or long (without feedback). There were four conditions based on the interval between the time of the keypress and the onset of the vibrotactile stimulus. In the $0 \mathrm{msec}$, no-delay condition, the vibration started as soon as the key press was detected. In the other three conditions, a delay of either 50,100 , or $150 \mathrm{msec}$ was inserted between the keypress and vibration onset (Fig. 11.3(b)).

Figure 11.4 shows the results for this experiment. The PSE for the no-delay condition is nearly identical to that observed in the self-initiation condition in our first vibrotactile 
experiment. Thus, the perceived duration of a self-initiated stimulus is lengthened even in comparison to an inferred standard duration (although this could also reflect a response bias in the second experiment). If this distortion is linked to the motor command, then we should observe that the effect will be enhanced when delays are introduced between the key press and stimulus onset. The results provide qualified support for this prediction. There is a 30-msec additional increase in the perceived duration during the 50-msec delay condition (evident by the shift of the PSE in the opposite direction), indicating that the estimate of stimulus onset is linked to a motor command triggering the stimulus. However, this effect is limited (see also Blakemore et al. 1999). There was no additional increase in perceived duration for the 100 -msec delay condition and for the 150 -msec delay condition, and perceived duration was similar to that observed in the no-delay condition. This nonmonotonicity suggests that when the delay becomes substantial, the contribution of the action-linked expectation is reduced and perceived duration is solely based on the stimulus.

In this section we discussed evidence indicating that such action commands may modify the perceived timing of sensory events. This influence on timing could be due to one of three nonexclusive mechanisms. First, an action may influence the perceived onset of the stimulus by altering the operation of an internal clock that marks the timing between events. Second, initiating an action may entail shifts in attention, and this could influence the perceived onset of a stimulus. Third, actions may modulate the perceptual processes, perhaps through the priming of perceptual channels by the motor commands, and thus advancing the time at which the stimulus onset is detected.

The model outlined in Fig. 11.3 does not differentiate between these hypotheses. Although all three mechanisms may be at play, there is some evidence to rule out the hypothesis that actions directly influence the perception of time. If action-linked changes in temporal perception were attributed to an adjustment in the rate of an internal clock, then the magnitude of this effect should also be affected by the duration of the stimulus being judged. However, Yarrow and colleagues have demonstrated that the magnitude of the chronostasis effect remains constant across a range of target stimulus durations (Yarrow et al. 2004). Thus, it does not appear that actions directly affect the internal representation of time itself.

We know from electrophysiological studies of the cat primary visual cortex that movements can directly influence principal sensory regions. The firing rates of V1 neurons increase during eye movements, even when these movements are made in the dark (Toyama et al. 1984). This saccade-related activity is also observed when the eye muscles are paralyzed, indicating that the $\mathrm{V} 1$ modulation is linked to the command to move the eyes rather than to the eye movements themselves. If we assume that an increase in baseline firing rate would lead to faster detection by bringing signal-induced firing closer to a decision threshold, then the perceived onset of a stimulus would occur earlier. This priming could be due to attentional shifts related to the movement command or direct corollary discharge from upstream motor regions. 
lengthened even in reflect a response or command, then introduced between for this prediction. the 50-msec delay indicating that the stimulus. However, additional increase l-msec delay condilay condition. This the contribution of solely based on the

mmands may modify be due to one of three onset of the stimulus ween events. Second, iuence the perceived 3ses, perhaps through is advancing the time

on these hypotheses. ience to rule out the uction-linked changes of an internal clock, ration of the stimulus that the magnitude of lus durations (Yarrow internal representation

sual cortex that moverates of V1 neurons de in the dark (Toyama teye muscles are parao move the eyes rather case in baseline firing ng closer to a decision ier. This priming could wect corollary discharge

\subsection{Integrating expectations and sensory input}

The preceding sections indicate that information linked to motor commands can influence perception of environmental events. We now turn to the question of how action- and sensory-based information is integrated.

5. There is emerging evidence that information between sensory modalities is integrated in a statistically optimal fashion (van Beers et al. 1999; Ernst \& Banks 2002; Kording \& Wolpert 2004). This integration process is unlikely to be limited to sensory information; internal, a priori expectations provide another salient source of information that should be combined with sensory signals (Weiss et al. 2002). It is reasonable to assume that the internal expectations linked to motor commands are combined with the sensory information in a similar, statistically optimal manner. In the remainder of this chapter we consider how this idea may help explain how a self-initiated movement influences the perceived duration of a stimulus triggered by that movement.

First let us assume that the perceived onset, P(onset) in Fig. 11.3(b), is the weighted contribution of sensory signals from the haptic system, $\mathrm{P}$ (haptic), and motor-linked expectations, $\mathrm{P}($ expect). Thus we can define the perceived onset as

$$
\mathrm{P}(\text { onset })=\omega_{\mathrm{H}} \mu_{\mathrm{E}}+\omega_{\mathrm{E}} \mu_{\mathrm{H}},
$$

where $\mu_{\mathrm{E}}$ and $\mu_{\mathrm{H}}$ represent the mean signals coming from the expectation and haptic channels, respectively. For the sake of simplicity we will assume that the mean of the expectation component occurs when the motor command is generated by the motor cortex and that the mean of the haptic component occurs when the sensory signal from the tactile stimulus is delivered to the sensory cortex in the brain. ${ }^{2}$ For a no-delay condition, the difference between $\mu_{\mathrm{E}}$ and $\mu_{\mathrm{H}}$ represents the delay introduced between the activation of motor cortex until the tactile information is registered. The terms $\omega_{H}$ and $\omega_{H}$ represent the weighted influence of each of these distributions on the resulting perceived onset. The relative weighted influence of each channel should be proportional to the overall variance such that

$$
\begin{aligned}
& \omega_{\mathrm{E}}=\left(1 / \sigma_{\mathrm{E}}^{2}\right) /\left(1 / \sigma_{\mathrm{E}}^{2}+1 / \sigma_{\mathrm{H}}^{2}\right) \\
& \omega_{\mathrm{H}}=\left(1 / \sigma_{\mathrm{H}}^{2}\right) /\left(1 / \sigma_{\mathrm{E}}^{2}+1 / \sigma_{\mathrm{H}}^{2}\right) .
\end{aligned}
$$

Thus, the lower the variability of a particular channel (i.e., the more reliable that channel's information capacity is), the stronger its influence will be on the resulting percept.

Using the observations from the experiment in which we varied the delay between the key press and stimulus, we now show how the influence of the motor-linked expectation on perception $\left(\omega_{\mathrm{E}}\right)$ can be estimated. To do this, we first want to understand how the perceived

${ }^{2}$ Which is equivalent to the time that the tactile stimulus is applied to the hand plus a constant delay in conduction time to the cortex. Because the delay would be equal for sensing both the onset and offset, this has little effect on the dynamics of our model. 
onset (relative to the key press) changes as a function of the delay. We define this as

$$
\Delta \mathrm{P}(\text { onset })=\omega_{\mathrm{H}} \mu_{\mathrm{E}}+\omega_{\mathrm{E}} \mu_{\mathrm{Hdelay}}-\left(\omega_{\mathrm{H}} \mu_{\mathrm{E}}+\omega_{\mathrm{E}} \mu_{\mathrm{H} 0}\right)
$$

where $\mu_{\text {Hdelay }}$ and $\mu_{\mathrm{H} 0}$ reflect the mean onsets estimated from the haptic sensory channel during the delay and no-delay conditions, respectively. Thus, $\Delta \mathrm{P}$ (onset) can simply be explained as

$$
\Delta \mathrm{P}(\text { onset })=\omega_{\mathrm{E}}\left(\mu_{\text {Hdelay }}-\mu_{\mathrm{H} 0}\right) .
$$

From this, the weighted influence of the motor-linked expectation can be defined as

$$
\omega_{\mathrm{E}}=\Delta \mathrm{P}(\text { onset }) / \Delta \mu_{\mathrm{H}},
$$

where $\Delta \mu_{\mathrm{H}}=\mu_{\mathrm{Hdelay}}-\mu_{\mathrm{H} 0}$. For example, in the 50-msec delay condition, $\Delta \mu_{\mathrm{H}}=50$. We now have a simple formula to estimate the influence of the internal expectation on perceived stimulus onset. ${ }^{3}$ Using the observed values in the delay experiment $\omega_{\mathrm{E}}=0.29$ when averaging across the three delay conditions, with considerable variation across the delay conditions $(50 \mathrm{msec}=0.58,100 \mathrm{msec}=0.25,150 \mathrm{msec}=0.02)$. By definition that average weight term for the sensory input is $\omega_{\mathrm{H}}=1-\omega_{\mathrm{E}}=0.71$. Thus, from this simplified example, it appears that the perceived onset is influenced to a greater degree by the actual onset of the tactile stimulus than by the movement-generated internal expectation of the onset.

One aspect of our results is at odds with the basic assumptions of this simple model. Contrary to what would be expected if the variance of the sensory channels and expectations were stationary, we found that perceived duration did not increase monotonically; there was no change for the 100-msec delay in comparison to the 50-msec delay, and it became shorter for the 150-msec delay. Within the framework of our model, this nonmonotonic pattern would reflect a change in the relative variances in either the haptic sensory channel or the motor-linked expectation. Assuming that the variance of the sensory channel remains constant over the different delays, at least for the range of values tested here, we would have to assume that the variance of the internal expectation increases for the longer delay conditions. This has the effect of "discounting" the internal expectation when there is a large mismatch between the timing of the expected and actual events. In the extreme, we would not expect any influence of the action if the stimulus onset occurred at an irregular time many seconds after the action. However, because the different delay conditions were randomly presented within the same blocks of trials, this effect cannot be explained by exposure to an "unreliable" expectation during longer delay trials.

There are several alternative accounts for the nonmonotonicity in the perceived duration function. First it could be that the integration of the sensory information and the expectation is a post hoc calculation, where the reliability of the expectation is revaluated prior to the generation of the final percept. This, however, fails to explain the electrophysiological

\footnotetext{
${ }^{3}$ In this simplified model, we assume that the mean offset from the haptic channel is veridical.
} 
define this as 10),

ptic sensory channel pnset) can simply be

can be defined as

ondition, $\Delta \mu_{\mathrm{H}}=50$. thernal expectation on xperiment $\omega_{\mathrm{E}}=0.29$ e variation across the $=0.02$ ). By definition 0.71 . Thus, from this to a greater degree by d internal expectation

of this simple model. innels and expectations notonically; there was , and it became shorter nonmonotonic pattern tic sensory channel or nsory channel remains tested here, we would es for the longer delay tation when there is a nts. In the extreme, we ccurred at an irregular delay conditions were annot be explained by

the perceived duration ion and the expectation revaluated prior to the he electrophysiological evidence that motor commands immediately influence primary sensory regions (Toyama et al. 1984) and would preclude a parsimonious account across the delay conditions. Another possibility is that the characteristics of the motor commands varied across the delay conditions. For example, the participants might have adopted a strategy of pressing the key until the vibration was felt despite our instructions to press the key in a quick and ballistic manner. Longer delays would result in more sustained presses, and this could influence the variance of the expectation generated from the motor command (e.g., if the variance of the expectation is linked to the velocity of the finger movement or the acceleration of the force of the key press). We did not measure key press duration in the current experiment and thus are unable to evaluate this hypothesis.

It is also possible that the perceived onset is not just a simple linear summation of an expectation and the sensory information but that this relationship is gain-modulated by a supervisory process that monitors the mean discrepancy between the two distributions (Blakemore et al. 2001). This would require the addition of a third parameter that represents the mean difference between the expected and sensed onset distributions. This new term would negatively weight the influence of the expectation distribution when there is a large discrepancy. Although such a model would more elegantly explain the data, it requires a supervisory process that monitors these discrepancies. Finally, it is entirely possible that the expectations are not integrated with sensory information in a linear fashion as outlined in this simple model. Regardless, the fact that the influence of action-linked expectations on perceived onset diminishes at longer delays makes it clear that more complicated mechanisms than simple statistical integration are at play during the perception of selfinitiated events.

\subsection{Summary}

Computational models of motor control have stressed that internal models must incorporate the anticipated sensory consequences of movements in the planning of the movements themselves (Jordan \& Rummelhart 1992). Our friendly waiter would not be able to do his job without being able to alter his movements based on such anticipatory processes. The emphasis in the current review is that expectations are not only important for modifying movement commands, but that these action-based signals also can influence perception. In the case of the waiter, we have shown that his perception of the weight of his tray is influenced by his own actions.

The second half of the chapter focused on one component of sensory expectations: how our actions influence the perceived time of resulting sensory events. Visual illusions such as chronostatis or the perceived duration in our vibrotactile experiments point to an influence of volitional actions on the perceived onset of sensory events initiated by those actions. Thus, one mechanism by which the action system influences perception is by influencing the perceived timing of sensory signals. The importance of timing in the generation of expectations is also evident in our hypothetical waiter example: the anticipatory response 
must be precisely timed. Reduce the force too early, and the postural hand will not be able to support the load. Wait until the sensory information is registered, and the load may become unstable due to the failure to reduce the supporting force.

These expectations, although adaptive, may also lead to systematic distortions of time. We introduced a simple model to capture these ideas, emphasizing how expectations and sensory information might be combined in our perception of sensory events with respect to our actions. The model provides a first pass at this integration problem, and we can already see serious limitations in its utility given the complex temporal relationship between the movements and perceived timing of the resulting stimuli. Nonetheless, it emphasizes the need to conceptualize the brain as a dynamic system in which movement- and sensoryoriented information are integrated in our perceptual experience.

\section{Acknowledgments}

We thank Joern Diedrishsen, Thom Griffiths, and Talia Konkle for helpful discussion.

\section{References}

Blakemore, S. J., Frith, C. D., \& Wolpert, D. M. (1999). Spatio-temporal prediction modulates the perception of self-produced stimuli. J Cogn Neurosci 11(5): $551-559$.

Blakemore, S. J., Frith, C. D., \& Wolpert, D. M. (2001). The cerebellum is involved in predicting the sensory consequences of action. Neuroreport 12(9): 1879-1884.

Blomdell, A., Bolmsjö, G., Brogårdh, T., Cederberg, P., Isaksson, M., Johansson, R., et al. (2005). Extending an industrial root controller - implementation and applications of a fast open sensor interface. IEEE Robotics \& Automation Magazine 12(3): 85-94.

Charpentier, A. (1891). Analyse experimentale de quelques elements de la sensation de poids. Archives de Physiologie Normales et Pathologiques 3: 122-135.

Diedrichsen, J., Verstynen, T., Hon, A., Lehman, S. L., \& Ivry, R. B. (2003). Anticipatory adjustments in the unloading task: is an efference copy necessary for learning? Exp Brain Res 148(2): 272-276.

Diedrichsen, J., Verstynen, T., Hon, A., Zhang, Y., \& Ivry, R. B. (2007). Illusions of force perception: the role of sensori-motor predictions, visual information, and motor errors. J Neurophysiol 97: 3305-3313.

Diedrichsen, J., Verstynen, T., Lehman, S. L., \& Ivry, R. B. (2005). Cerebellar involvement in anticipating the consequences of self-produced actions during bimanual movements. J Neurophysiol 93(2): 801-812.

Dufossae, M., Hugon, M., \& Massion, J. (1985). Postural forearm changes induced by predictable in time or voluntary triggered unloading in man. Exp Brain Res 60: 330-334.

Ernst, M. O., \& Banks, M. S. (2002). Humans integrate visual and haptic information in a statistically optimal fashion. Nature 415(6870): 429-433.

Haggard, P., Clark, S., \& Kalogeras, J. (2002). Voluntary action and conscious awareness. Nat Neurosci 5(4): 382-385.
Hodinott-Hil hanging

Hugon, M., I induced Arch 39.

Jordan, M., \& teacher.

Kording, K. I Nature ‘

Lacquaniti, F organizé

Lum, P. S., R Feedfor' 172-18(

Masson, J., I anticipal patholog

Matin, L., Pis (1982). humans

Nelson, R. (1 sensorin

Nijhawan, R.

Oliver, M., V Somatos Neurosc

Park, J., Schl duration

Schmidt, W. Psych: I

Sharma, J., D acquisit 1758-1

Soechting, J. in threeNeurosc

Toyama, K., of eye $n$

van Beers, $R$ finger is Res 125

Weiskrantz, I oneself.

Weiss, Y., Si percepts

Welch, R. B. Kaufma New Yo 
and will not be ad the load may

ortions of time. xpectations and with respect to we can already hip between the emphasizes the t- and sensory-

discussion

orediction

11(5):

s involved in 379-1884.

ansson, R., et al. applications of 12(3): 85-94. sensation de 5.

3). Anticipatory learning? Exp

Ilusions of force and motor

ellar

is during

induced by

in Res 60:

information in a cious awareness.
Hodinott-Hill, I., Thilo, K. V., Cowey, A., \& Walsh, V. (2002). Auditory chronostasis: hanging on the telephone. Curr Biol 12(20): 1779-1781.

Hugon, M., Massion, J., \& Wiesendanger, M. (1982). Anticipatory postural changes induced by active unloading and comparison with passive unloading in man. Pflugers Arch 393(4): 292-296.

Jordan, M., \& Rumelhart, D. (1992). Forward models: supervised learning with a distal teacher. Cog Sci 16: 307-354.

Kording, K. P., \& Wolpert, D. M. (2004). Bayesian integration in sensorimotor learning. Nature 427(6971): 244-247.

Lacquaniti, F., Soechting, J. F., \& Terzuolo, C. A. (1982). Some factors pertinent to the organization and control of arm movements. Brain Res 252(2): 394-397.

Lum, P. S., Reinkensmeyer, D. J., Lehman, S. L., Li, P. Y., \& Stark, L. W. (1992). Feedforward stabilization in a bimanual unloading task. Exp Brain Res 89: $172-180$.

Masson, J., Ioffe, M., Schmitz, C., Viallet, F., \& Gantcheva, R. (1999). Acquisition of anticipatory postural adjustments in a bimanual load-lifting task: normal and pathological aspects. Exp Brain Res 128(1-2): 229-235.

Matin, L., Picoult, E., Stevens, J. K., Edwards, M. W. Jr., Young, D., \& MacArthur, R. (1982). Oculoparalytic illusion: visual-field dependent spatial mislocalizations by humans partially paralyzed with curare. Science 216(4542): 198-201.

Nelson, R. (1996). Interactions between motor commands and the somatic perception in sensorimotor cortex. Curr Opin Neurobiol 6: 801-810.

Nijhawan, R. (1994). Motion extrapolation in catching. Nature 370(6487): 256-257.

Oliver, M., Verstynen, T., \& Ivry, R. B. (2003). Did I Do That? - Modulating the Somatosensory Percept through Self Production. Poster presented at the Cognitive Neuroscience Society meeting, 2003.

Park, J., Schlag-Rey, M., \& Schlag, J. (2003). Voluntary action expands perceived duration of its sensory consequence. Exp Brain Res 149(4): 527-529.

Schmidt, W. C. (2000). Endogenous attention and illusory line motion reexamined. $J$ Exp Psych: Hum Percept Perform 26: 980-996.

Sharma, J., Dragoi, V., Tenenbaum, J. B., Miller, E., \& Sur, M. (2003). V1 neurons signal acquisition of an internal representation of stimulus location. Science $\mathbf{3 0 0}$ : $1758-1763$.

Soechting, J. F., Lacquaniti, F., \& Terzuolo C. A. (1986). Coordination of arm movements in three-dimensional space. Sensorimotor mapping during drawing movement. Neuroscience 17: 295-311.

Toyama, K., Komatsu, Y., \& Shibuki, K. (1984). Integration of retinal and motor signals of eye movements in striate cortex cells of the alert cat. J Neurophysiol 51: 649-665.

van Beers, R. J., Sittig, A. C., \& Denier van der Gon, J. J. (1999). Localization of a seen finger is based exclusively on proprioception and on vision of the finger. Exp Brain Res 125(1): 43-49.

Weiskrantz, L., Elliott, J., \& Darlington, C. (1971). Preliminary observations on tickling oneself. Nature 230(5296): 598-599.

Weiss, Y., Simoncelli, E. P., \& Adelson, E. H. (2002). Motion illusions as optimal percepts. Nat Neurosci 5(6): 598-604.

Welch, R. B., \& Warren, D. H. (1986). Intersensory interactions. In K. R. Boff, L. Kaufman, \& J. P. Thomas (eds.), Handbook of Perception and Human Performance. New York: Wiley. 
Yarrow, K., Haggard, P., Heal, R., Brown, P., \& Rothwell, J. C. (2001). Illusory perceptions of space and time preserve cross-saccadic perceptual continuity. Nature 414(6861): 302-305.

Yarrow, K., Haggard, P., \& Rothwell, J. C. (2004). Action, arousal, and subjective time. Conscious Cogn 13(2): 373-390.

Yarrow, K., \& Rothwell, J. C. (2003). Manual chronostasis: tactile perception precedes physical contact. Curr Biol 13(13): 1134-1139. 